\title{
OPC-TDM network performance improvement by the use of full-scalable optical packet compression/ decompression units
}

\author{
Slaviša Aleksić, Kemal Bengi, Vjeko Krajinović \\ Vienna University of Technology, Institute of Communication Networks \\ Favoritenstrasse 9/388, A-1040,Vienna, Austria \\ e-mail:Slavisa.Aleksic@tuwien.ac.at
}

Key words: Optical packet compression/decompression, OTDM, performance study

Abstract: An optical packet compression/decompression scheme applicable in ultrahighspeed OTDM networks is proposed. This full-extensible scheme allows high compression rates and large packet sizes by relaxing the medium access constrains. The improvement of the Optical Packet Compression-TDM (OPCTDM) network performance achieved by reducing the rate-conversion latency is shown.

\section{INTRODUCTION}

Optical Packet Compression-Time Division Multiplexing (OPC-TDM) networks provide ultrahigh-speed access to the optical medium employing the optical packet compression/decompression technique [1,2]. Fig. 1 shows an OPC-TDM access node architecture. Depending on the packet destination address, incoming high-speed packets can be either dropped or forwarded by an optical switch located at the node. An optical delay line is employed in the optical path to compensate the header processing latency. The essential part of an OPC-TDM access node is the Optical Packet Compression/ Decompression Unit, responsible for rate-conversion of ultra high-speed optical data streams to lover rate data streams that can be detected, saved and processed electronically. On the other hand, the low bit-rate packet acquired from the transmitting queue must be at first up-converted to the high 
medium data rate and after that inserted in the network by replacing of a dropped or an empty packet.

Recently, several methods for optical rate conversion (compression and decompression) have been proposed [3,4,5]. Most of these optical rate conversion schemes are based on an optical buffer (optical storage loop) and a sampling technique. However, there are some restrictions concerning bitrate and packet size if this technique is used. Those restrictions are caused by difficulties in storage of ultrahigh-speed large optical packets for a larger period because of the optical signal impairing caused by dispersion and ASE (Amplified Spontaneous Emission) noise accumulation during amplification. A further technique for optical packet compression is a feed-forward delayline structure consisting of $q=\log _{2}(n)$ stages reported in [5]. This technique allows simultaneous compression and decompression of $n$-bit large optical packets using the same device. It's simple to build by using only passive components, but for larger packet lengths the splitting losses ( $3 \mathrm{~dB}$ per stage) must be compensated with an optical amplifier. However, the maximal packet size $L_{p, \max }$ is limited by the compression rate $K$ as follows:

$$
L_{p, \max } \leq K-1 \quad ; \quad K=\frac{T_{0}}{\tau_{0}}
$$

Consequently, for an 100:1 compression $(K=100)$ the $L_{p, \max }$ is limited to 99 bits. Such short packets are usually impractical in many applications.

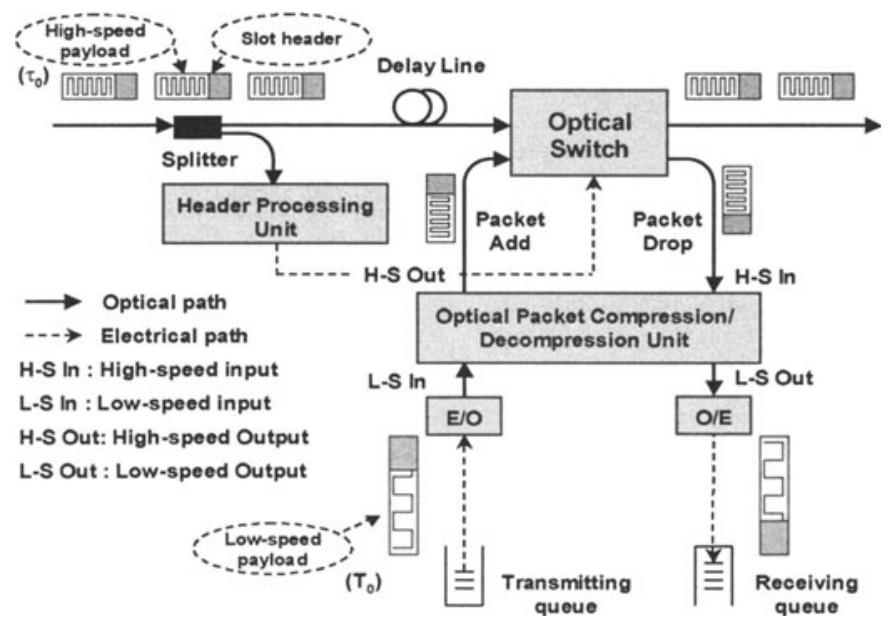

Fig. 1 OPC-TDM access node architecture.

This paper is organized as follows. In Section 2 of this paper, we propose a full scalable optical packet compression/decompression scheme using a parallel arrangement of Optical Delay Line Lattices (ODLLs). This scheme allows simultaneously compression and decompression of optical 
packets not limited in size and compression rate. In Section 3, a network performance analysis is performed in order to investigate the impact of this scheme on the network performance, especially on the average packet delay.

\section{OPTICAL PACKET COMPRESSION/DECOMPRESSION UNIT}

A figure-of-merit for OPC-TDM networks is the packet rate-conversion time relative to the packet length $\left(\alpha=T_{p r d} / T_{p}\right)$. In the case of a packet compressor consisting of a parallel arrangement of MODLLs the $\alpha$ is given by:

$$
\alpha=\frac{T_{p r c}}{T_{p}}=\frac{K-1}{M}
$$

The $\alpha$ signifies the number of packets in the network not accessible by a receiving node caused by down-conversion latency. In other words, the receiver must first down-convert the received packet, before it is ready for receiving another packet. For a conversion rate $K=100$ and by the use of only one ODLL for the rate conversion $(M=1)$, the receiver can access only each $100^{\text {th }}$ packet in the network. This hard restriction leads to an inefficient bandwidth utilization.

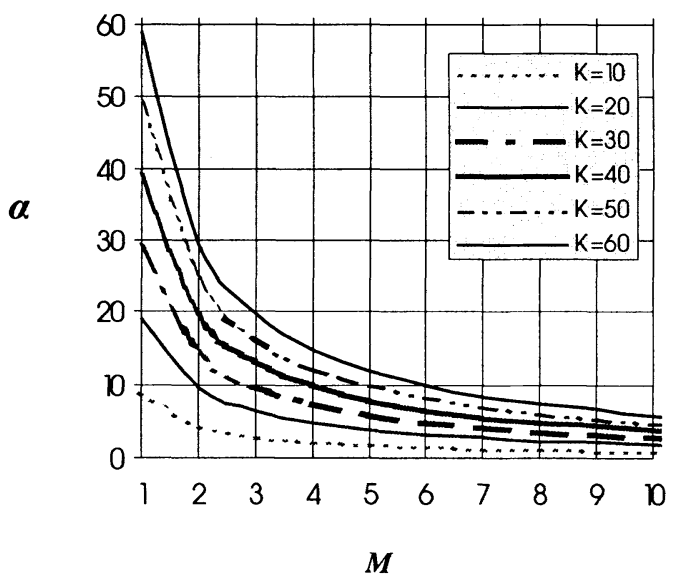

Fig. 2 Packet down-conversion time relative to packet length.

The impact of compression rate, packet size, and the number of deployed parallel transceivers on the OPC-TDM ring network performance has been partly investigated $[2,6]$. The results show that decreasing the $\alpha$ significantly 
improves network performance. Fig. 2 plots $\alpha$ over the number of deployed parallel ODLLs related to the different compression rates $(K)$. The $\alpha$ shows a gradual decrease if $M$ increases. For example, an additional ODLL causes a decreasing of $\alpha$ value from 49 to 24.5 for a compression rate $K=50$. Therefore, we propose and investigate a packet compression/decompression scheme using a parallel arrangement of delay lines, which provides lower $\alpha$ values for high compression rates and large packet sizes.

The proposed scheme depicted in Fig. 3 consists of $M$ parallel compression/decompression units, each of them responsible for rateconversion of a part of the optical packet. The operation of the device can be described as follows: high-speed input packet (H-S In) is divided into $M$ successive $n$-bit sequences using a splitter and $M$ bi-directional opticál gates. Those sequences are then copied $n$ times in a $n$-bit ODLL. Each copy is delayed with respect to the next copy of the packet by $T_{0}-\tau_{0}$ as shown in Fig. 4(a). An ultrafast OTDM demultiplexer selects within very narrow switching window bits spaced at the bit period $\mathrm{T}_{0}$, whereby the down-conversion of the high-speed sequence is done. Finally, all $M$ sequences are delayed by an appropriate optical delay line $\mathrm{DL}_{2, m}$ and combined by a $M \times 1$ coupler. Thereby, the whole high-speed input packet is down-converted.

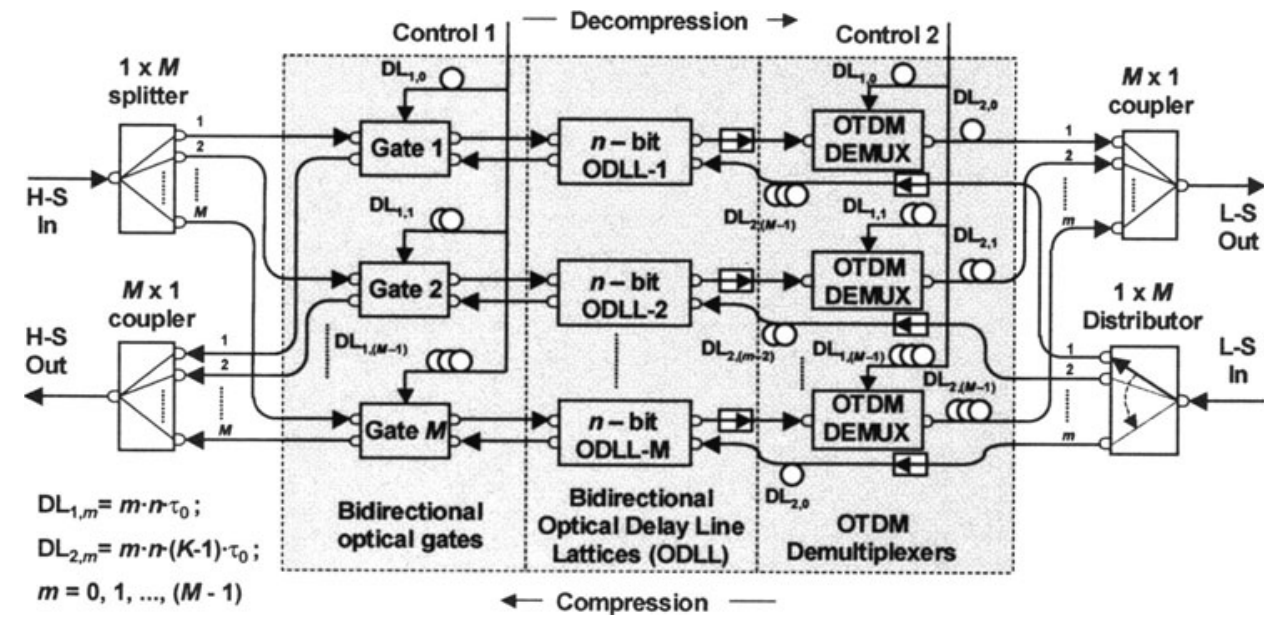

Fig. 3 Optical packet compression/decompression unit.

Moreover, using the same device in the reverse direction, a low-speed packet (bit period $T_{0}$ ) can be compressed as shown in Fig. 4(b). The lowspeed optical packet (L-S In) is first divided into $M$ successive sequences by an $1 \times M$ distributor. Each sequence is then delayed by an appropriate delay line $\mathrm{DL}_{2, \mathrm{~m}}$ and compressed by an $n$-bit ODLL. The fully compressed sequences are finally selected by the gates and combined by a $M \times 1$ coupler. 
The result is the high-speed output signal (bit period $\tau_{0}$ ) that has the same bit pattern sequence as the low-speed packet. Note that both compression and decompression can occur simultaneously in the same device.

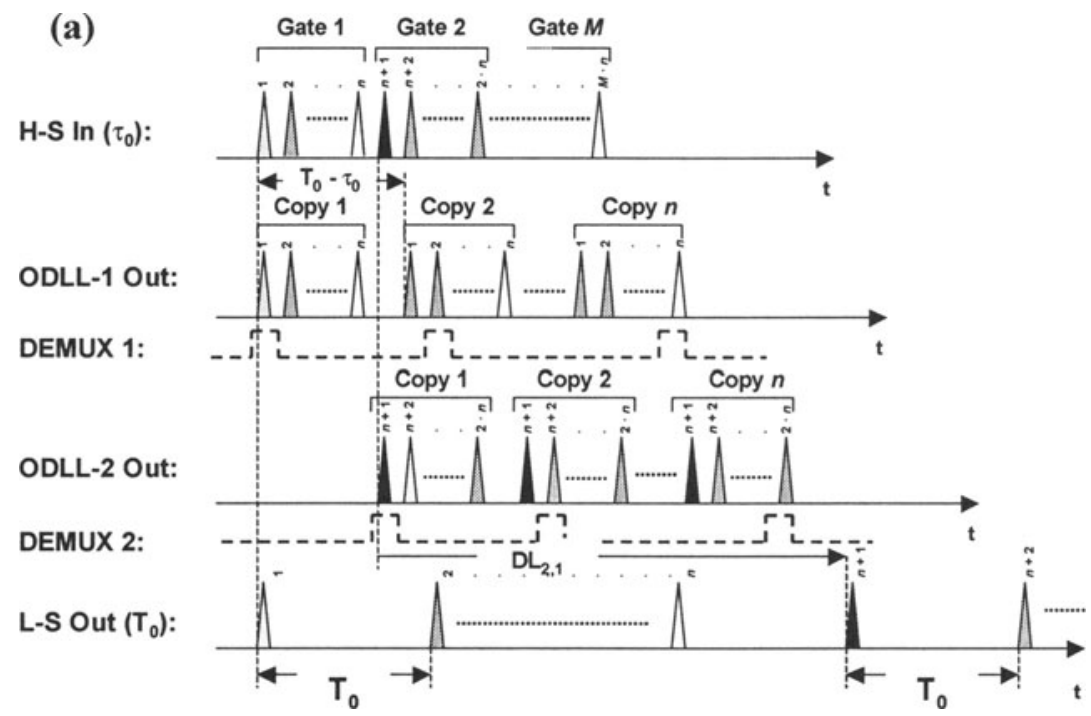

(b)

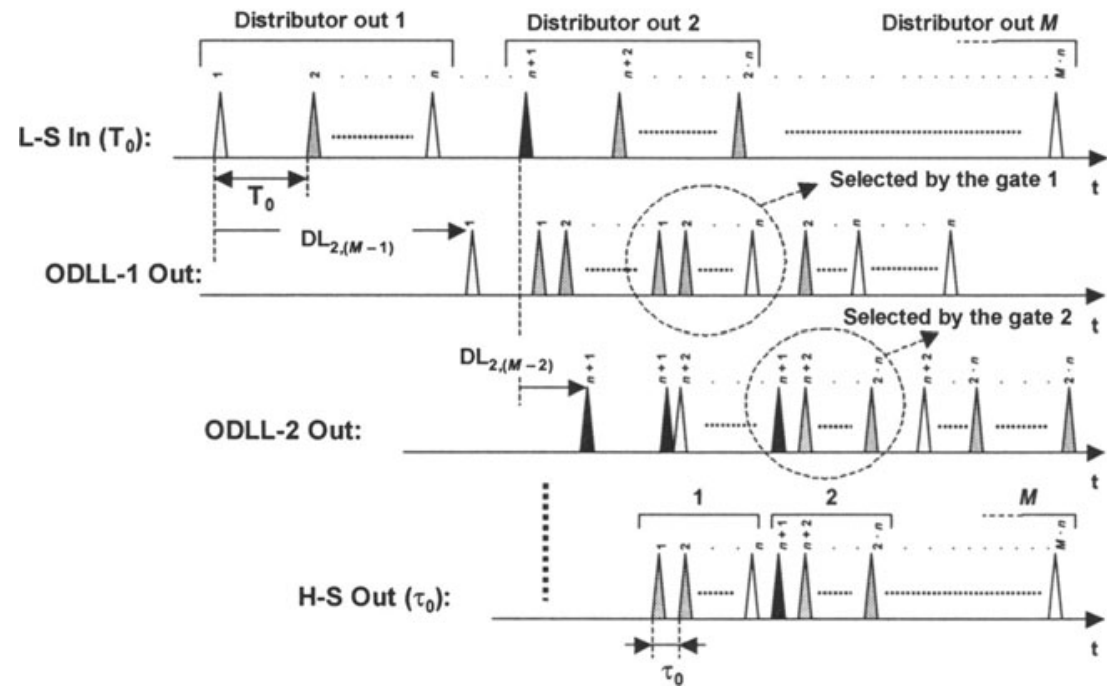

Fig. 4 Timing chart of the optical packet (a) decompression and (b) compression operation

Simulations are carried out in order to investigate the feasibility of the proposed optical packet compression/decompression scheme. The simulation setup is shown in Fig. 5(a). 


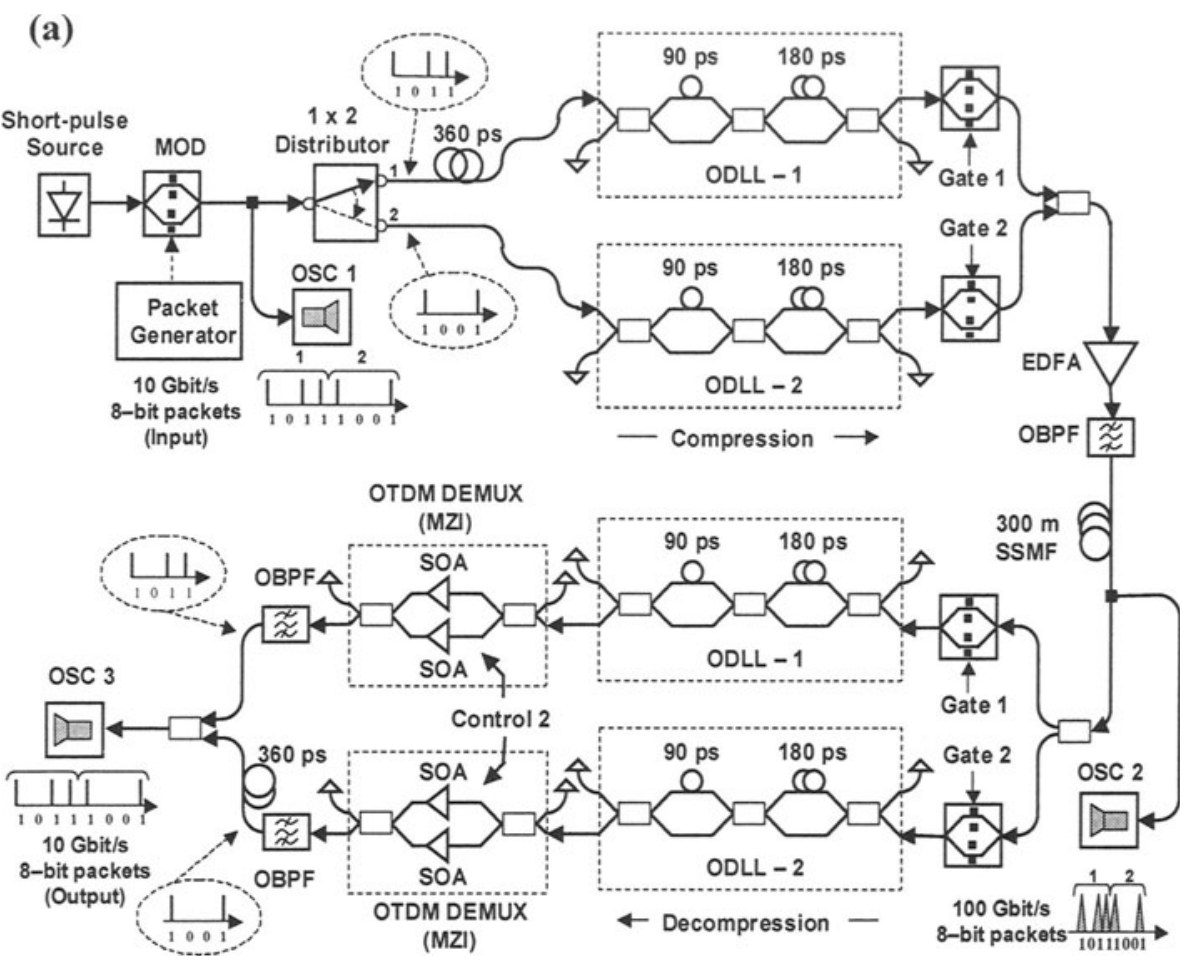

(b)

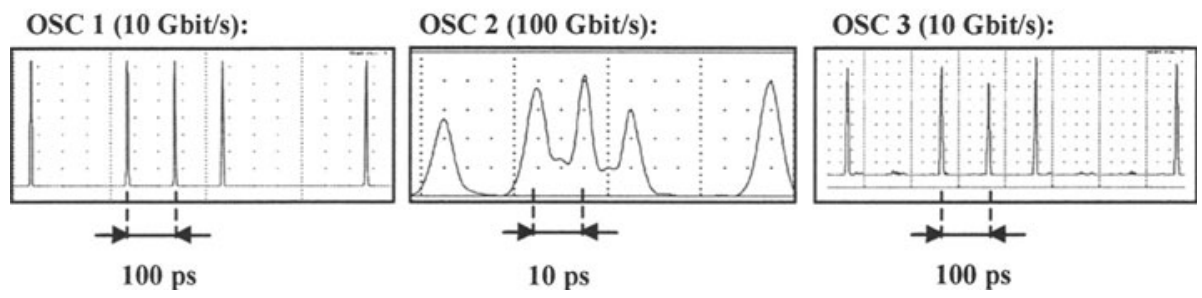

Fig. 5 Simulation setup and signal traces

8-bit large packets at $10 \mathrm{Gbit} / \mathrm{s}$ (4 ps FWHM pulses at $1.55 \mu \mathrm{m}$ wavelength) were generated and compressed by a compressor composed of two parallel 4-bit ODLLs. The compressed packets were then transmitted over $300 \mathrm{~m}$ of standard single mode fiber (SSMF) and finally decompressed by the receiver using the proposed scheme. In the transmission path an EDFA (Erbium Doped Fiber Amplifier) and an OBPF (Optical Band Pass Filter) were deployed for fiber loss compensation and amplifier ASE removing respectively. A symmetric Mach-Zehnder Interferometer (MZI) with SOAs in its arms was used as an OTDM demultiplexer. An 168 to 10.5 Gbit/s demultiplexing experiment has already been reported using a similar 
structure [7]. We used a $\mathrm{LiNbO}_{3}$ modulator for implementing the gating functionality. A fast all-optical switch (e.g. MZI) can be also deployed if ultra-fast gates are required. A $10 \mathrm{Gbit} / \mathrm{s}$ input packet (OSC 1), a $100 \mathrm{Gbit} / \mathrm{s}$ optical signal after SSMF (OSC 2), and decompressed $10 \mathrm{Gbit} / \mathrm{s}$ packet by receiver (OSC 3) are shown in Fig. 5(b). The signal traces show the general feasibility of the proposed scheme, where in our case an extinction ratio better than $13 \mathrm{~dB}$ was measured at the low-speed output (OSC 3). Note that an important advantage of this scheme is its scalability. It is possible to choose an optimal configuration (optimal $M$ and $n$ values) for achieving a lower $\alpha$ value along with large packet sizes and large compression rates. In comparison with a compression/decompression unit consisting of only one $n$-bit ODLL (requiring $q=\log _{2}(n)$ stages), the compression/decompression unit consisting of $M$ ODLLs can be realized using ODLLs with only $q=$ $\log _{2}(n / M)$ stages for compression of $n$-bit large packets. In this case a small number of stages implies less splitting losses. Thereby, optical signal amplification needs not to be applied.

\section{OPC-TDM NETWORK PERFORMANCE STUDY}

In this Section, a mathematical performance study is performed in order to investigate the impact of the proposed optical packet compression/ decompression scheme on the network performance. In this performance study, particular attention is paid to the comparison of the proposed scheme with a single feed-forward delay-line structure considering average packet transfer delay.

\subsection{Protocol Description}

We assume a system based on the slotted synchronized single-ring architecture as shown in Fig. 6. The MAC-protocol used in this system is simple and based on the Slotted Ring scheme with destination release and spatial slot reuse. Since only every $(\alpha+1)$-th slot is accessible by the receiving node (due to the strict time constraints associated with the packet rate-conversion time), the slots are grouped into frames. Each slot within a frame is dedicated to a receiving station on the ring. The frame size is then $N$ $=(\alpha+1)$ slots, where $N$ denotes the number of nodes in the system. The length of the time slots is a design issue and in our studies we set the slot length to the average message length in the system. 


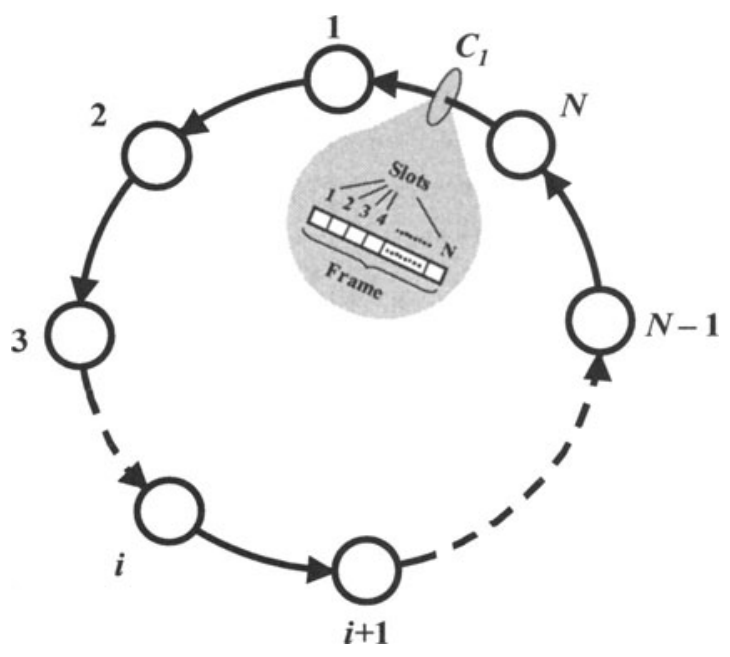

Fig. 6 Considered OPC-TDM single-ring network

\subsection{Performance Study}

In this section, a preliminary performance study of the considered slotted ring network concerning different packet compression/decompression unit realizations is performed.

\subsubsection{Average Packet Transfer Delay}

Let us assume that the packets arrive at each transmitting queue with a Poisson arrival process. Further, each of $N$ slots in a frame is uniquely assigned to a destination station forming a receiver-oriented TDMA system. As we know, in TDMA the transmission capacity of the medium $C$ is divided into $N$ portions (channels), each with the capacity equal to $C / N$. Moreover, we assume a uniform traffic, i.e. a packet is equally likely to be transmitted to any of the $N-1$ other stations. The average packet transfer delay is defined as the expected delay in transmitting a message from any source node to any destination node including packet transmission time $\left(T_{p}\right)$, waiting time in the queue ( $\left.T_{\text {queue }}\right)$, and end-to-end transmission time $(\tau)$ of the network. For a slotted-ring OPC-TDM network it will also include the packet rate-conversion time $\left(T_{p r c}\right)$, and the time a node must wait for a free slot assigned to corresponding destination node $\left(T_{\text {access }}\right)$. Therefore, the average packet transfer delay in our case is given by:

$$
D=T_{p}+T_{\text {prc }}+T_{\text {queue }}+T_{\text {access }}+\tau
$$


The packet transmission time $\left(T_{p}\right)$ corresponds to the time it takes to transmit a data packet of $X$ bits at $R$ bit/s data transmission rate. The packet rate-conversion time $\left(T_{p r c}\right)$ is given above by Equation (2). The time a packet must wait in the transmitting queue including the waiting time for the $i$-th slot assigned to the destination node $i$ is given by [8]. Considering the frame synchronisation period along with the mean waiting time of the $M / D / 1$ queue model, we obtain:

$$
T_{\text {queue }}=\frac{N}{2} T_{p}+\frac{N}{2} T_{p} \frac{\rho}{1-\rho}=\frac{N}{2(1-\rho)} T_{p}
$$

If the $i$-th slot assigned to the destination node $i$ is already busy, the transmitting node must wait for the next frame. To find the time a packet must wait for a free slot assigned to the destination node $i$, we determine a probability $P_{i}$, which denotes the probability that the slot $i$ within the frame is busy. For overloaded network with uniform traffic and destination release the probability $P_{i}$ is given by $P_{i}=(N-2) /(2 \cdot(N-1))$. Thus $T_{\text {access }}$ can be expressed as:

$$
T_{\text {access }}=P_{i} \cdot T_{\text {frame }}=\frac{N-2}{2(N-1)} N \cdot T_{p}
$$

Using the expression (3), combining $T_{p r c}=(N-1) \cdot T_{p}$, and the results of (4), and (5) we obtain the average packet transfer delay as:

$$
D=\frac{T_{p} N}{2}\left(2+\frac{1}{1-\rho}+\frac{N-2}{N-1}\right)+\tau
$$

Finally, $N$ in (6) can be replaced by $K$ and $M$ using $N=(\alpha+1)$ and (2) then gives companion expression to (6) for $D$, namely:

$$
D=T_{p} \frac{M+K-1}{2 M}\left(3+\frac{1}{1-\rho}-\frac{M}{K-1}\right)+\tau
$$

Curves of $D$ versus offered load $\rho$ related to different number of the parallel ODLLs $(M)$ are plotted in Fig. 7. Fig. 8 shows the average packet transfer delay in dependence on the number of slots in the frame related to different network loads. For all plotted curves we assumed a compression 
rate of $K=100$, a packet length of $T_{p}=10 \mathrm{kbits} \cdot 10 \mathrm{ps}=0.1 \mu \mathrm{s}$ (at 100 $\mathrm{Gbit} / \mathrm{s}$ ), and a $20 \mathrm{~km}$ fiber-ring network with $\tau \approx 97 \mu \mathrm{s}$.

From the plots it can be observed that adding additional ODLLs can reduce the average packet delay. If the offered load increases, the differences between the curves increase as well. The significant improvement can be seen for $M=2$. Another advantage of the proposed packet compression/ decompression scheme is decreasing the frame length. Particularly, for higher load $(>0.8)$ the average packet delay increases significantly if the frame length increases.

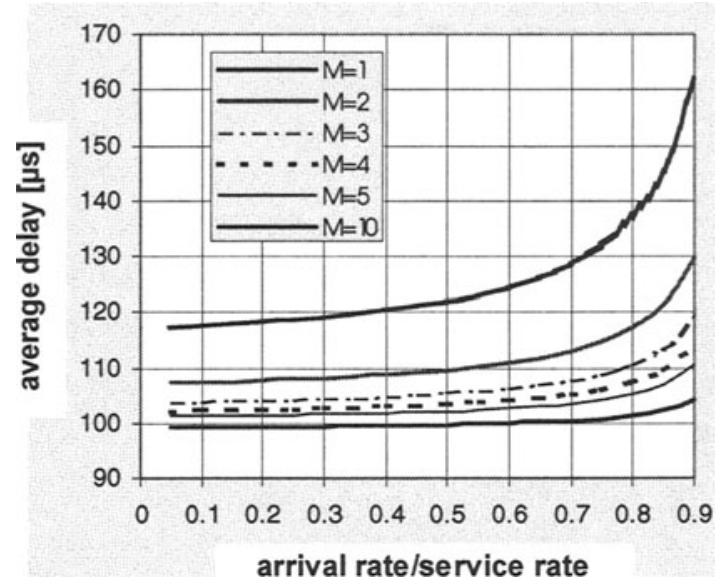

Fig. 7 Average delay vs. offered load related to $M$

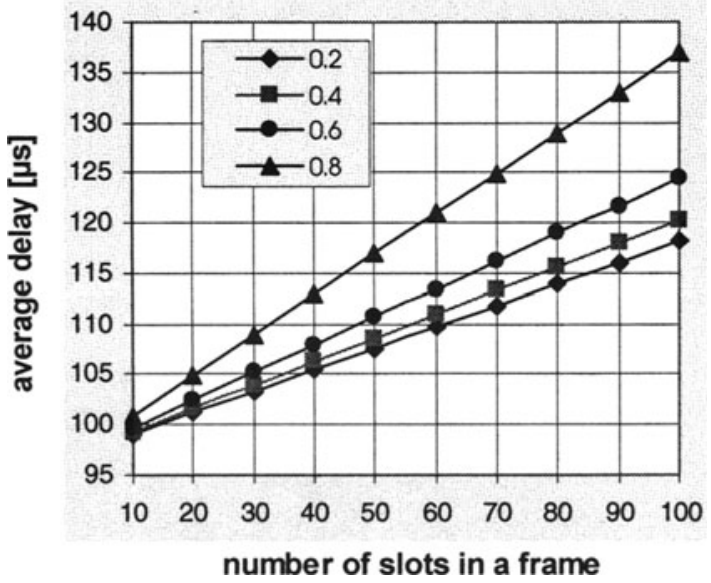

Fig. 8 Average delay vs. number of slots in the frame related to offered load 


\section{CONCLUSION}

In conclusion, an optical packet compression/decompression scheme using a parallel arrangement of optical delay line structures is proposed and investigated in this paper. The proposed scheme allows high compression rates and large packet sizes by reducing the medium access constrains. Our simulations show feasibility of the proposed scheme. The impact of the proposed scheme on the network performance is theoretically investigated. Particularly, the average packet transfer delay in dependence on the number of deployed optical delay line lattices (ODLLs) is shown. A significant performance improvement in comparison with a single feed-forward delayline structure can be achieved by adding additional ODLLs.

\section{ACKNOWLEDGEMENTS}

This work is supported by the Austrian Science Fund FWF (Fonds zur Förderung der wissenschaftlichen Forschung) under contract P13144-INF. The tool PTDS was employed for the simulations.

\section{REFERENCES}

[1] S. W. Seo, K. Bergman, P. R. Prucnal, "Transparent Optical Networks with Time Division Multiplexing”, IEEE JSAC, Vol. 14, No. 5, (1996), pp. 1039 - 1051.

[2] K.Bengi, S.Aleksic, "Design Considerations for a Slotted OTDM Ring-LAN", in NOC2000 (Stuttgart, 2000), pp. $191-198$.

[3]H. Toda, F. Nakada, M.Suzuki, A. Hasegawa, „An Optical Packet Compressor Using a Fiber Loop for a feasible all optical TDM Network", in ECOC'99, (Nice, France,1999), pp. I-256 - I-257.

[4] N. S. Patel, K. L. Hall, K. A. Rauschenbach, "Optical Rate Conversion for High-Speed TDM Networks”, IEEE Photon. Techn. Letters, Vol. 9, (1997), pp.1277 - 1279.

[5] P.Toliver, K.L. Deng, I. Glesk, P.R. Prucnal, "Simultaneous Optical Compression and Decompression of $100 \mathrm{~Gb} / \mathrm{s}$ OTDM Packets Using a Single Bidirectional Optical Delay Line Lattice", IEEE Photon. Techn. Letters, Vol. 11, (1999), pp. 1183 - 1185.

[6] K. Gokyu, K. Baba, M. Murata, "Path Setup Algirithms for Uniform/Nonuniform Traffic in Unidirectional Optical Compression TDM Rings", in OptiComm 2000 (Dallas, Texas, October $22-26,2000$ ), pp. $98-109$.

[7] S. Nakamura, et al, " 168 Gbps error-free demultiplexing with hybrid-integrated symmetric Mach-Zehnder all-optical switch", in OFC2000 (Baltimore, Maryland, 2000), pp. ThF3-1 - ThF3-3.

[8] J.Spragins, J. Hammond, and D. Pawlikowski, "Telecommunications: Protocols and Design”, Addison-Wesley Publishing Co., Reading, MA, 1991. 